New Media

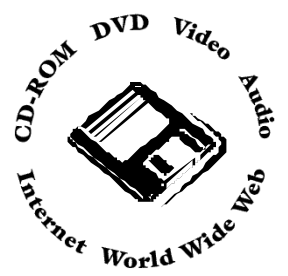

\section{Review of Atlas of Anesthesia on CD-ROM. Volume IV}

Principles of Anesthetic Techniques and Anesthetic Emergencies

Harcourt Brace and Co. Ltd. \$130 (US). ISBN 0443-07507-7

This is the fourth volume of a series of CD-ROMs under the overall editorial guidance of Dr. Ronald D. Miller. This particular volume is edited by Dr. Kevin K. Tremper.

The underlying engine used is Folio Bound VIEWS, a popular but aging system often used for CD-ROM publishing in the medical community. The whole CD-ROM takes only $227 \mathrm{MB}$, so it presumably could be copied to your hard drive without difficulty should the need arise. I had no trouble installing the program and running it on an old $133 \mathrm{MHz}$ Pentium computer running Windows 95.

Essentially, this product is a series of graphic images with associated figure legends. Beside each figure legend is a thumbnail image on to which one clicks to obtain a full graphic image. Unfortunately, the figure legends are lost when the expanded figure is put into view.

Another drawback to the CD-ROM is that the images are not accessible as individual files, making it difficult to incorporate them in your own teaching presentations. As far as I can see, the only way of incorporating the images into your own presentations is by using screen capture techniques. While many of the illustrations are superbly prepared, some of them are of particularly poor quality. For example, Figure 9A in Chapter 7 contains film sprocket marks that should have been deleted by cropping. Another problem is that the tables are presented as graphic images rather than as text and consequently are not searchable.

Finally, for an atlas that purported to deal with anesthetic emergencies, there was too little material on the topic of emergency management of the airway, one of the most common problems we deal with in clinical practice. While there are nice sections concerning aspiration, anterior mediastinal masses and the management of epiglottis, the sections on the ASA difficult airway algorithm and management of the cannot intubate, cannot ventilate scenario were notable by their absence.

Despite its limitations, I expect that the atlas will play a useful role in medical education. However, I expect that many individuals will find the paper version of the atlas more fun to leaf through.

For completeness, listed below are the titles of all the volumes comprising the series:

Volume I. Critical Care

Volume II. Scientific Principles of Anesthesia

Volume III. Preoperative Preparation and Intraoperative Monitoring

Volume IV. Principles of Anesthetic Techniques and Anesthetic Emergencies

Volume V. Subspecialty Care

Volume VI. Pain Management

Volume VII. Pediatric Anesthesia

Volume VIII. Cardiothoracic Anesthesia

D. John Doyle MD PHD FRCPC

Toronto, Ontario

\section{Speech Recognition Comes of Age}

I can remember when I was a student in my early university days, years before the advent of microprocessors and personal computers, wondering whether or not I would live long enough to witness speech recognition software that would work. I dreamed of software that would allow one to dictate directly to a computer. (A victim of a traditional college preparatory school thinking, I had neither chance nor motivation to learn to type in high school. I still type painfully using only two fingers.)

I took my first plunge into speech recognition technology about five years ago, buying a package offered by IBM that would only operate under OS $/ 2$. After an enormous amount of technical effort on the part of both myself and IBM we got the system to work on a 486 laptop but the performance was so poor it was 
still much easier to type with two fingers. About two years later, I bought an upgrade to the product and ran it on a faster computer, only to find that the performance was still unacceptable. More recently, I tried once again, this time getting the latest release of Dragon Naturally Speaking, Version 4, and ran it on an even faster computer. Once again, there were severe performance problems, mostly related to the volume of word inaccuracies. I was ready to give up.

Then it occurred to me that I might get better performance if I used a USB microphone instead of using an ordinary analog microphone plugged into the computer sound card. Apparently, there is a lot of electrical noise inside most computers, so that analog signals can become heavily contaminated before being processed. The problem is even worse with laptop computers, with their crowded electronics. However, this problem can be eliminated (at least on latest generation computers) by using a USB microphone. (USB stands for "universal serial bus" and is a new interface technology supported by Windows 98 that can be used for hooking up peripheral components to computers. USB technology can be used for printers, scanners, microphones, TV cameras, and even keyboards, mice and modems.)

With the addition of a USB microphone from Telex, a remarkable transformation occurred to my system. The first thing I noticed, after installing the USB microphone, was that vocabulary training took only a few minutes, and not the better part of an hour that I was accustomed to. Secondly, the performance of the system was finally good enough to use for real world dictation tasks. The accuracy of my system is now good enough to dictate real letters and write real reports, even with the noise of the hard drive and fan in the background. (In fact I can even get reasonable results with the TV playing gently in the background).

Yes, speech recognition has arrived and is finally practical. It is still imperfect, but it is definitely now worth while to two-finger typists. I can see that it will not be long before speech recognition technology will be available for clinical data entry during routine anesthesia care. Still, it is not clear that this technology will be sufficiently reliable in the noisy operating room environment that it will see widespread adoption for use in charting and documentation.

D. John Doyle MD PHD FRCPC

Toronto, Ontario

\section{Patient's Use of the Internet}

Now that Internet access has become more commonly available, some patients are using this technology to assist in their medical care. The following story illustrates this point.

A middle-aged man noted that for several years food would get caught in what he believed to be a pouch in his esophagus. Although usually not a problem, he noted with increasing frequency that filling of the pouch would sometimes lead to vomiting in socially embarrassing circumstances. Not having a family doctor, he sought information on his condition via friends, relatives and the Merck Manual. Suspicious that the description of Zenker's diverticulum given there matched his condition, he then did a search over the Internet (using the Lycos search engine), and came upon a reference to Zenker's diverticulum in an article posted on GASNet. As a result, the patient contacted the author (DJD) by telephone seeking clinical advice. This information was provided informally, outlining what investigations might be done and providing the patient with the names of some clinicians known to be experienced in esophageal conditions.

This story illustrates some of the novel ways in which patients may sometimes acquire clinical information. As the Internet continues to increase in popularity, patients with Internet access will "surf the net" for information about their condition and be able to readily compare different management options. UseNet, discussion groups, and organization websites (e.g. the American Cancer Society) will allow those suffering from clinical conditions to receive medical information and even emotional support for themselves, their families and friends from those affected by the same medical problem. The impact of the Internet on public health can be enormous.

Patients, especially those from the United States, can also use the Internet to locate area physicians (e.g. through the American Medical Association website), check their credentials, and can even access disciplinary reports, malpractice judgments, and arbitration awards on a particular physician. Such data are often provided in the United States through state licensing authorities. One U.S. website (www.docboard.org), maintained by Administrators in Medicine (AIM), has links to 15 state regulatory bodies, including some osteopath and podiatry licensing bodies. Local magazines in many major U.S. cities (e.g. Minneapolis St. Paul Magazine, Boston Magazine) have published their surveys of best area doctors, often grouped by specialty. In Canada, the websites of licensing organizations will provide lists of member doctors, including those who are accepting 